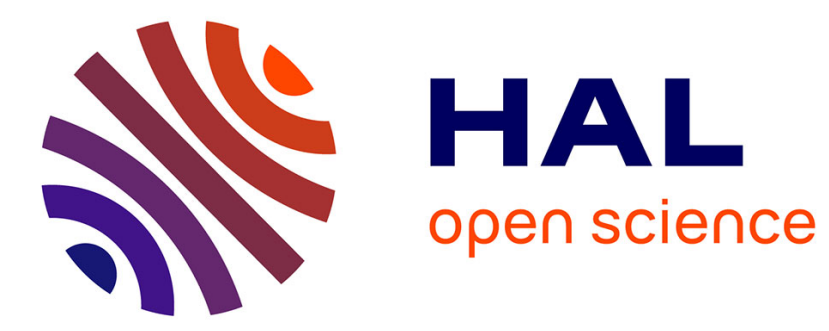

\title{
Excitation Transfer in Functionalized Carbon Nanotubes
}

Gurvan Magadur, Jean-sébastien Lauret, Valérie Alain-Rizzo, Christophe

Voisin, Philippe Roussignol, Emmanuelle Deleporte, Jacques A. Delaire

\section{To cite this version:}

Gurvan Magadur, Jean-sébastien Lauret, Valérie Alain-Rizzo, Christophe Voisin, Philippe Roussignol, et al.. Excitation Transfer in Functionalized Carbon Nanotubes. ChemPhysChem, 2008, 9, pp.1250. hal-00288315

\section{HAL Id: hal-00288315 https://hal.science/hal-00288315}

Submitted on 16 Jun 2008

HAL is a multi-disciplinary open access archive for the deposit and dissemination of scientific research documents, whether they are published or not. The documents may come from teaching and research institutions in France or abroad, or from public or private research centers.
L'archive ouverte pluridisciplinaire HAL, est destinée au dépôt et à la diffusion de documents scientifiques de niveau recherche, publiés ou non, émanant des établissements d'enseignement et de recherche français ou étrangers, des laboratoires publics ou privés. 


\section{Excitation transfer in functionalized} carbon nanotubes

\author{
G. Magadur, ${ }^{[a, b]}$ J.S. Lauret, ${ }^{\left[{ }^{[a]}\right]}$ V. Alain-Rizzo, ${ }^{[b]}$ \\ C. Voisin, ${ }^{[c]} \mathrm{Ph}$. Roussignol, ${ }^{[\mathrm{c}]}$ E. Deleporte ${ }^{[\mathrm{a}]}$ and \\ J. A. Delaire ${ }^{[b]}$
}

Tailoring the properties of carbon nanotubes by functionalizing their side-wall is one of the key challenges towards the realization of carbon nanotube-based optoelectronic devices [1, 2]. This technique aims at combining the remarkable transport properties of the tube $[3,4]$ with the versatility of the optical properties of organic molecules. In this study, we achieved " $\pi$-stacking" functionalization of nanotubes with hydrosoluble porphyrins $[5,6]$. These complexes show a total quenching of the porphyrin fluorescence, while the photoluminescence of the nanotubes is enhanced when the excitation is tuned in resonance with the Soret band of the porphyrins. This is the evidence for an efficient excitation transfer within the nanotube/porphyrin complex, which corresponds to the implementation of a key functionnality: an organic graft acting as an antenna and the nanotube acting as a collector.

Synthesis of carbon nanotubes/ organic molecules complexes certainly represents a new way to obtain bulk samples of nanotube-based materials with uniform optical properties. In this way, interactions between nanotubes and porphyrin molecules have attracted much attention for about five years in connection with applications such as nanotubes debundling [7], nanotube sorting [6], photovoltaic cells [8-11] or biology [2]. Most of the studies deal with either covalent $[9,11]$ or " $\pi$ - stacking" [58 , 12] functionalization. Changes in the porphyrin optical absorption and emission spectra in the presence of nanotubes have been reported $[7-9,12]$ while other studies show no changes $[5,6]$. In contrast and despite the great interest of the question, little work has been devoted to the alteration of nanotubes properties. In this Letter, optical absorption, photoluminescence and photoluminescence excitation spectroscopies are used to investigate these modifications and the overall properties of the complex. We show that porphyrins hardly perturbe the key electonic properties of the nanotubes and realize two functions: they act both as a surfactant for nanotube

[a] Mr G. Magadur, Dr J.S. Lauret, Pr E. Deleporte

Laboratoire de Photonique Quantique et Moléculaire, Institut d'Alembert

ENS Cachan

61 avenue du Président Wilson 94235 Cachan Cedex, France.

Fax: (+) 33147405567

E-mail: lauret@lpqm.ens-cachan.fr

[b] Dr V. Alain-Rizzo, Pr J. Delaire

Laboratoire de Photophysique et Photochimie Supramoléculaires et Macromoléculaires, Institut d'Alembert ENS Cachan

61 avenue du Président Wilson 94235 Cachan Cedex, France.

[c] Dr C. Voisin, DrPh. Roussignol

Laboratoire Pierre Aigrain

Ecole Normale Supérieure

24 rue Lhomond, 75005 Paris, France debundling and solvatation and as an antenna transferring the optical excitation to the nanotube.
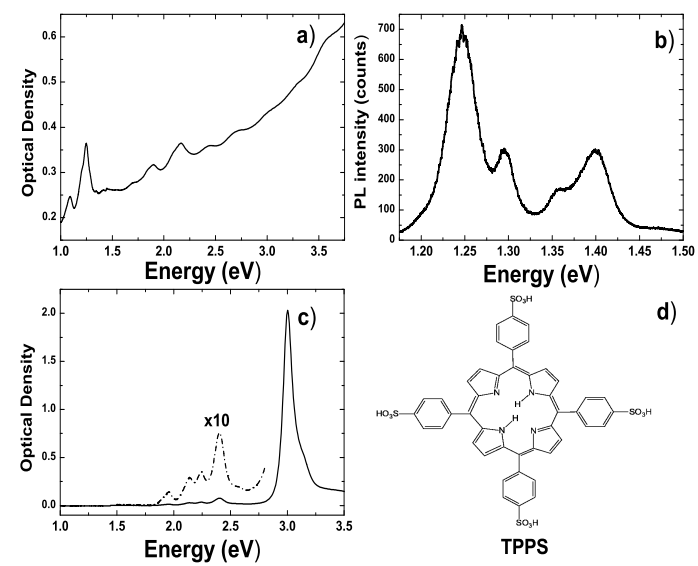

Figure 1. a) Optical absorption of CoMoCat nanotubes embedded in Sodium Dodecyl Sulfate micelles in a pH 8 Normadose buffer. b) Corresponding photoluminescence spectrum of CoMoCat nanotubes embedded in micelles (excitation energy: $2.331 \mathrm{eV}$ ). c) Optical absorption of TPPS in a pH 8 Normadose buffer (dashed curve: $Q$ bands contribution rescaled for clarity). d) Chemical structure of TPPS. The phenyl groups are not in the plane of the macrocycle because of the steric hindrance.

In this study, we use CoMoCat carbon nanotubes which are single-wall nanotubes (SWNT) with a mean diameter of about $0.8 \mathrm{~nm}$ [13]. The nanotubes are functionalized with the 5,10,15,20-tetrakis(4-sulfonatophenyl) porphyrin (TPPS). The optical absorption spectrum of CoMoCat nanotubes embedded in Sodium Dodecyl Sulfate (SDS) micelles at $\mathrm{pH} 8$ is displayed in figure 1a). The corresponding PL spectrum excited at $2.331 \mathrm{eV}$ is shown in figure $1 b$ ). Four main emission bands are observed at $1.248 \mathrm{eV}, 1.295 \mathrm{eV}, 1.357 \mathrm{eV}$ and $1.399 \mathrm{eV}$, which are commonly attributed to the $(6,5),(8,3),(9,1)$ and $(6,4)$ nanotubes respectively $[13,14]$. The porphyrin optical absorption spectrum is displayed in figure 1c). One can observe the weak $Q$ absorption bands (in the 1.7-2.5 eV range) and the prominent Soret band (at $3 \mathrm{eV}$ ). The PL spectrum (grey curve in figure $2 \mathrm{~b}$ )) exhibits two bands at $1.77 \mathrm{eV}$ and $1.91 \mathrm{eV}$ corresponding to singlet-singlet transitions. Note that, once rescaled, the optical absorption spectra of TPPS are identical for concentrations between $4 \%$ and $96 \%$ [15], showing that porphyrins do not aggregate up to this concentration.

The absorption spectrum of nanotube/porphyrin suspensions is shown in figure 2a) for several porphyrin concentrations. The dark dashed curve represents the free porphyrin $(4 \%)$ absorption spectrum rescaled for clarity. For porphyrin concentrations below $72 \%$, the Soret band peaks at $2.88 \mathrm{eV}$ which corresponds to a red shift of $120 \mathrm{meV}$ with respect to the absorption band of free porphyrins. Since we have checked that no aggregation of the porphyrin occurs for these 
concentrations, we assign this red shift to the interaction between the porphyrin molecules and the nanotubes.

For a concentration of $72 \%$ and up, a shoulder appears at 3 $\mathrm{eV}$, which corresponds to the Soret band of free porphyrins. This means that nanotubes no longer bind to porphyrins above this concentration and that additional porphyrins remain free in the suspension. Further experiments are under progress to determine more accurately the saturation threshold. Note that we cannot observe the $Q$ bands of nanotube/porphyrin complexes since the absorption is mainly due to the nanotubes in this energy range (see figures 1a) and 1c)).

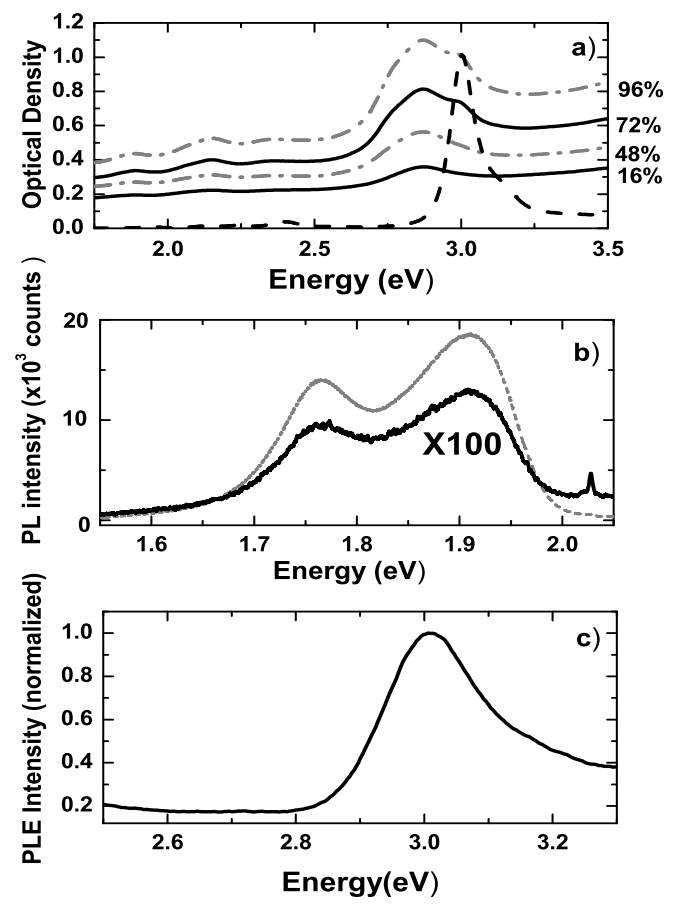

Figure 2. a) Optical absorption of TPPS/CoMoCat nanotubes suspensions around the Soret band energy for porphyrin concentrations ranging from $16 \%$ to $96 \%$. Free porphyrin ( $4 \%$ ) absorption spectrum (black dashed curve) rescaled for clarity). b) Photoluminescence spectra of TPPS (4\% [15]) alone (grey) and in the presence of nanotubes (black). c) Photoluminescence excitation spectrum of a nanotube/porphyrin suspension detected at $1.77 \mathrm{eV}$.

Figure $2 \mathrm{~b}$ ) shows the photoluminescence spectra (excited at $3.06 \mathrm{eV})$ of free porphyrins (4\%, grey line) and nanotube/porphyrin complexes (4\%, black curve). The latter shows a quenching of $99 \%$ of the porphyrin line (around $1.8 \mathrm{eV}$ ) indicating a strong interaction between " $\pi$-stacked" porphyrins and nanotubes. The photoluminescence excitation spectrum of a nanotube/porphyrin suspension (detection at $1.77 \mathrm{eV}$ ) is shown in figure 2c). It peaks at $3 \mathrm{eV}$, that is at the energy of the Soret band of the free porphyrin. Moreover, after rescaling this spectrum is exactly the same as the one of free porphyrin (not shown here). We conclude that the weak remaining photoluminescence signal of the porphyrins actually arises from residual free porphyrins. As a consequence we deduce that " $\pi$-stacked" porphyrins no longer emit light, supporting the assumption of a strong nanotubeporphyrin interaction.
Several mechanisms may account for the red shift of the Soret band. Protonation of TPPS leads to a red shift of about $140 \mathrm{meV}$ [16]. Since the experiments reported here are performed in a $\mathrm{pH} 8$ buffer and since the TPPS pKa are about 5, protonation of the porphyrin can be ruled out. A red shift of the Soret band may also stem from the formation of $J$-aggregates [8, 12], which is generally observed in acid environments and leads to a red shift of about $500 \mathrm{meV}$. We tried to aggregate TPPS in the $\mathrm{pH} 8$ buffer by increasing gradually the porphyrin concentration without any success. In contrast, in an acid medium we were able to form $\mathrm{J}$ aggregates with this protocol, in agreement with previous studies [16]. Thus we deduce that $\mathrm{J}$-aggregates cannot exist in our experimental conditions and therefore cannot account for the observed red shift.

A red shift of the Soret band has also been reported for porphyrins laid on a substrate [17] or self-assembled into nanoparticles [18]. In that case the molecules flatten to enhance the interaction with the substrate or with other porphyrin molecules. The phenyl groups, which make a $90^{\circ}$ angle with the plane of the macrocycle for free porphyrins in solution, tend to tilt and to reduce this angle when interacting with the substrate or other molecules. This behavior leads to a red shift of the Soret band of about $200 \mathrm{meV}$ for a tilt of $30^{\circ}$ [17]. In our experiments, we assign the observed red shift of the Soret band to a flattening of the porphyrin in contact with a nanotube.

As a consequence, this red shift of the Soret band should depend on the nanotube diameter; in fact, the larger the nanotube diameter, the more the porphyrin has to flatten in order to optimize the $\pi-\pi$ interactions with the nanotube. Thus, the red shift should increase with the tube diameter [17]. Preliminary results show that TPPS adsorbed on nanotubes with a mean diameter of $1.2 \mathrm{~nm}$ (synthesized by the electric arc method) exhibit a $170 \mathrm{meV}$ red shift of the Soret band. Compared to the previously mentioned CoMoCat nanotubes, we observe a $40 \%$ increase of the red-shift for a $50 \%$ increase of the nanotube diameter, which is qualitatively consistent with our interpretation.

Nevertheless, it has been shown that the photoluminescence signal of porphyrins is preserved when they flatten to form nanoparticles [18]. Thus, the strong quenching of the porphyrin fluorescence in the presence of nanotubes cannot be due to the deformation of the molecules. This rather seems to indicate a strong electronic interaction between porphyrins and nanotubes.

Optical absorption and fluorescence were recorded similarly in the spectral range of the nanotube first excitonic transitions usually labelled " $S_{11}$ ". The evolution of the nanotube absorption as a function of the porphyrins concentration is depicted in figure 3a). We first observe that the specific absorption band of the nanotubes is better resolved when the porphyrin concentration is higher. In order to be more quantitative, we define an aspect ratio "R" such as:

$$
R=\frac{O D_{\max }}{O D_{\min }}
$$

where $O D_{\max }$ is the optical density at the maximum of the band and $O D_{\min }$ is the optical density at the high energy foot $(1.32 \mathrm{eV})$ of the band. We get $R=1.09$ for a porphyrin concentration of $8 \%$ and $R=1.37$ for a concentration of $72 \%$. For comparison, for nanotubes embedded in micelles (reference material, see figure

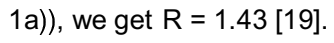


The absorption band consists of a shoulder at $1.19 \mathrm{eV}$ and a maximum at $1.251 \mathrm{eV}$ (for TPPS concentration up to $72 \%$ ). For higher porphyrin concentrations, the shoulder disappears (see the $96 \%$ curve in figure 3a)) while the absorption at $1.251 \mathrm{eV}$ increases. This seems to indicate that TPPS interacts preferentially with some specific types of nanotubes (i.e. chiralities).

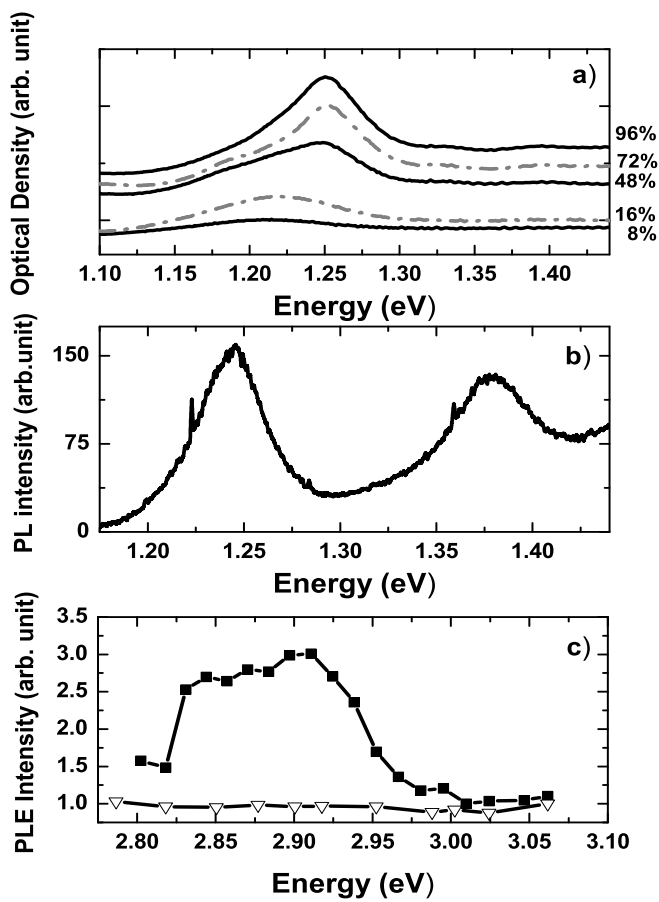

Figure 3. a) Optical density of TPPS/CoMoCat nanotubes suspension around first excitonic transition of nanotubes for different concentrations of porphyrins (from $8 \%$ to $96 \%$ ). Curves have been arbitrary shifted on the $\mathrm{Y}$-axis for clarity. b) Photoluminescence signal of CoMoCat nanotubes in presence of TPPS excited at $2.331 \mathrm{eV}$. c) Photoluminescence excitation spectra of a nanotube/porphyrin suspension (black squares) and of nanotubes embedded in $\mathrm{SDS}$ micelles (open triangles) detected at $1.246 \mathrm{eV}$.

Further insight can be gained by photoluminescence measurements. Single wall carbon nanotubes consisting essentially of surface atoms, their properties are very sensitive to their environment [20-22]. Regarding their optical properties, it is well known that the luminescence is strongly quenched when nanotubes are aggregated in bundles $[21,22]$ or in contact with a surface [23]. Since only isolated nanotubes luminesce [21, 24], the observation of some PL signal for nanotubes/TPPS suspensions (see Figure $3 b$ )) ensures that some individual nanotubes have been functionalized and that the interaction with the porphyrin molecules is "soft" in contrast to covalent grafting which suppresses the luminescence [11]. This means that nanotubes intrinsic properties are essentialy preserved in nanotube/porphyrin complexes. Moreover, if porphyrins actually foster the debundling of nanotubes, one expects an enhancement of the quantum efficiency of the suspensions when more tubes are functionalized [21, 22, 24, 25]. Indeed, we qualitatively observe that the higher the porphyrin concentration, the larger the nanotubes PL intensity.

The PL signal of functionalized nanotubes exhibits two bands at $1.246 \mathrm{eV}$ and $1.389 \mathrm{eV}$. Compared to the PL signal of nanotubes embedded in surfactant, two bands -corresponding to $(8,3)$ and $(9,1)$ nanotubes- are missing in the spectrum of functionalized nanotubes. This is consistent with our previous conclusion that a partial nanotube sorting is achieved.

Moreover, the PLE spectrum of nanotube/porphyrin complexes detected at $1.246 \mathrm{eV}$ (see figure 3c)) shows that the $\mathrm{PL}$ signal of the functionalized nanotubes is greatly enhanced when the sample is excited at $2.88 \mathrm{eV}$. In contrast, the PLE spectrum of nanotubes embedded in SDS micelles is flat in this spectral range (see figure $3 \mathrm{c}$ )). We assign this PLE band at $2.88 \mathrm{eV}$ to the Soret band of the " $\pi$-stacked" porphyrins, as shown from the absorption spectra (see figure 2a)). This clearly means that the PL signal arises from functionalized nanotubes. Furthermore, it shows that light absorbed on " $\pi$-stacked" porphyrin states results in a population on excited states of nanotubes. Therefore, it brings evidence for an efficient excitation transfer from the porphyrins to the nanotubes within the " $\pi$-stacked" complexes. Finally, we have realized an hybrid material combining the absorption of the porphyrin molecules and the luminescence of nanotubes where the transfer of excitation is very efficient.

In summary, we have demonstrated the functionalization of CoMoCat nanotubes by TPPS porphyrins. Porphyrins " $\pi$-stacked" on nanotubes exhibit a red shift of the Soret band, which has been interpreted in terms of a flattening of the porphyrin molecule in order to optimize the $\pi-\pi$ interactions with the nanotube. Moreover, we have shown that TPPS interacts preferentially with some specific classes of nanotubes. We report that the photoluminescence signal of functionalized nanotubes is preserved. In the same time, " $\pi$-stacked" porphyrins no longer emit light, but the nanotube fluorescence is enhanced when the excitation energy is tuned in resonance with the absorption band of the porphyrins bringing evidence for a strong excitation transfer from TPPS to the nanotubes. This opens avenues to applications in the field of nanotube based opto-electronic devices. In view of potential applications, the understanding and the optimization of this excitation transfer would be valuable. Time-resolved experiments are under progress to investigate further the electronic states involved in this coupling, as well as its nature and its dynamics.

\section{Experimental Section}

The porphyrin molecules were synthesized according to conventional procedures described in the literature [27]. It is soluble in a pH 8 Normadose buffer $\left(10^{-2} \mathrm{M}\right)$ and its chemical structure is depicted in figure 1d). Nanotubes functionalization is achieved by adding a porphyrin solution $\left(1 \mathrm{mg} \cdot \mathrm{mL}^{-1}\right)$ in a suspension of SWNT $\left(0.1 \mathrm{mg} \cdot \mathrm{mL}^{-1}\right)$ in a pH 8 Normadose buffer. The porphyrin weight fraction is adjusted and increased from $4 \%$ (porphyrin/SWNT w/w) up to $96 \%$. After porphyrin addition, the solution is sonicated for $3 \mathrm{~h}$ with a dismembrator. The sample is placed in a thermostat and cooled in ethanol at $1^{\circ} \mathrm{C}$ during sonication.

Optical absorption spectra are recorded with a spectrophotometer (lambda 900 Perkin-Elmer). Two cw-laser diodes at $3.062 \mathrm{eV}(405 \mathrm{~nm})$ and $2.331 \mathrm{eV}(532 \mathrm{~nm})$ are used as excitation sources for photoluminescence (PL) experiments. The $\mathrm{PL}$ signal is dispersed in a spectrograph (Spectrapro 2500i, Roper Scientific) and detected by a Si CCD camera (Pixis 100B, PI Acton). When detecting in the visible range, 
photoluminescence excitation experiments (PLE) are performed with a spectrofluorometer (Jobin-Yvon Fluoromax-3), while for detection in the near infrared range a tunable frequency doubled Ti:Saphire laser is used as the excitation source $(2.75 \mathrm{eV}$ $3.1 \mathrm{eV}$ ).

\section{Acknowledgements}

The authors are grateful to I. Leray for providing the porphyrin molecules and to D.E. Resasco for providing the CoMoCat nanotubes. LPQM, PPSM and LPA are "Unités mixtes" de recherche associées au CNRS (UMR8537; UMR8531; UMR8551). This work was supported by the GDR-E "nanotube" (GDRE2756) and grant "C'Nano IdF Phototube" from "Région lle de France".

We thank one of the reviewers for bringing to our attention the highly related work published online by J.P. Casey et al. [27].

\section{Keywords: (carbone nanotubes · functionalization · optical} properties $\cdot$ photoluminescence $\cdot$ porphyrin)

[1] M.S. Strano, C.A. Dyke, M.L. Usrey, P.W. Barone, M.J. Allen, H. Shan, C. Kittrell, R.H. Hauge, J.M. Tour, R.E. Smalley, Science 2003, 301, 15191522.

[2] D.M. Guldi, J. Phys. Chem. B 2005, 109, 11432-11441.

[3] P. Avouris, Z. Chen, V. Pereibeinos, Nature nanotechnology 2007, 2, 605 615

[4] A.D. Mohite, P. Gopinath, H.M. Shah, B.W. Alphenaar, Nano Lett. 2008, 8, 142-146.

[5] H. Murakami, T. Nomura, N. Nakashima, Chem.Phys. Lett. 2003, 378, $481-$ 485.

[6] H. Li, B. Zhou, Y. Lin, L. Gu, W. Wang, K.A. Shiral Fernando, S. Kumar, L.F. Allard, YP. Sun J. Am. Chem. Soc. 2004, 126, 1014-1015.

[7] G.M. Aminur Rahman, D.M. Guldi, S. Campidelli, M. Prato, J. Mater. Chem. 2006, 16, 62-65

[8] T. Hasobe, S. Fukuzumi, P.V. Kamat, J. Phys. Chem. B 2006, 110, $25477-$ 25484.

[9] M. Alvaro, P. Atienzar, P. De la Cruz, J.L. Delgado, V. Troiani, H. Garcia, F. Langa, A. Palkar, L. Echegoyen, J. Am. Chem. Soc. 2006, 128, 6626-6635.

[10] C. Ehli, G.M. Aminur Rahman, N. Jux, D. Balbinot, D.M. Guldi, F. Paolucci, M. Marcaccio, D. Paolucci, M. Melle-Franco, F. Zerbetto, S. Campidelli, M. Prato, J. Am. Chem. Soc. 2006, 128, 11222-11231.
[11] M.A. Herranz, N. Martin, S. Campidelli, M. Prato, G. Brehm, D. M. Guldi, Angew. Chem. Int. Ed. 2006, 45, 4478-4482.

[12] T. Hasobe, S. Fukuzumi, P.V. Kamat, J. Am. Chem Soc. 2005, 127 11884.

[13] G. Lolli, L. Zhang, L. Balzano, N. Sakulchaicharoen, Y. Tan, D.E. Resasco, J. Phys. Chem. B 2006, 110, 2108-2115.

[14] S.M. Bachilo, M.S. Strano, C. Kittrell, R.H. Hauge, R.E. Smalley, R.B. Weisman, Science 2002, 298, 2361-2366.

[15] In order to compare quantitatively suspensions with and withou nanotubes, we label free porphyrin suspensions with the expression " $\mathrm{X} \%$ ". It corresponds to the porphyrin concentration that would be used in a $\mathrm{X} \%$ nanotube/porphyrin suspension where $\mathrm{X}$ represents the ratio porphyrin/SWNT w/w for an initial NT concentration of $0.1 \mathrm{mg} \cdot \mathrm{mL}^{-1}$.

[16] O. Ohno, Y. Kaizu, H. Kobayashi J. Chem. Phys. 1993, 99, 4128-4139.

[17] Z. Chernia, D. Gilla, Langmuir 1999, 15, 1625-1633.

[18] Z. Ou, H. Yao, K. Kimura, J. of Photochem. And Photobiol. A: Chemistry 2007, 189, 7-14.

[19] The authors want to point out that, even if the absolute value of "R" is subject to uncertainty, the general trend is reproducible indicating that porphyrins help solubilizing nanotubes, even if the solubilizing efficiency is not as high as for surfactants.

[20] J. S. Lauret, C. Voisin, S. Berger, G. Cassabois, C. Delalande, Ph. Roussignol, L. Goux-Capes, A. Filoramo, Phys. Rev. B 2005, 72, 113413

[21 M.J. O'Connell, S.M. Bachilo, C.B. Huffman, V.C. Moore, M.S. Strano, E.H. Haroz, K.L. Rialon, P.J. Boul, W.H. Noon, C. Kittrell, J. Ma, R.H. Hauge, R.B. Weisman, R.E. Smalley Science 2002, 297, 593-596.

[22] J.S. Lauret, C. Voisin, G. Cassabois, C. Delalande, P. Roussignol, L. Capes, O. Jost, Phys. Rev. Lett. 2003, 90, 057404.

[23] J. Lefebvre, Y. Homma, P. Finnie, Phys. Rev. Lett. 2003, 90, 217401.

[24] J.S. Lauret, C. Voisin, G. Cassabois, Ph. Roussignol, C. Delalande, A Filoramo, L. Capes, E. Valentin, O. Jost, Physica E 2004, 21, 1057-1060.

[25] S. Berger, C. Voisin, G. Cassabois, C. Delalande, P. Roussignol, X. Marie Nano Lett. 2007, 7, 398-402.

[26] A.D. Adler, F.R. Longo, J.D. Firanelli, J.D. Goldmacher and J.A. Assur, J. Org. Chem. 1967, 32, 476-476.

[27] J.P. Casey, S.M. Bachilo, R.B. Weisman, J. Mat. Chem. 2008, DOI: $10.1039 / b 716649 d$ 